\title{
The Epidemiology of Influenza in Children Hospitalized at the Hospital of Sikasso, Mali 2015-2019
}

\author{
Assetou Cissouma ${ }^{1 *}$, Madou Traoré2 ${ }^{2}$ Guédiouma Dembélée ${ }^{3}$, Seydou Diarra ${ }^{4}$, \\ Adama Mamby Keita4, Abdoulaye Kissima-Traoré5, Djibril Kassogué6, \\ Soumaila Alama Traoré7, Flagnouma Diallo1, Dade Ben Sidi Haidara1, Samba Sow ${ }^{4}$ \\ ${ }^{1}$ Pediatric Department, Sikasso Hospital, Sikasso, Mali \\ ${ }^{2}$ Department of Medicine and Infectious Disease, Sikasso Hospital, Sikasso, Mali \\ ${ }^{3}$ Pediatric Department, Mali Hospital, Bamako, Mali \\ ${ }^{4}$ Centre for Vaccine Development-Mali (CVD-Mali), Bamako, Mali \\ ${ }^{5}$ Cardiology Department, Sikasso Hospital, Sikasso, Mali \\ ${ }^{6}$ Pediatric Department, Timbuktu Hospital, Timbuktu, Mali \\ ${ }^{7}$ Gynecology and Obstetrics Department of Sikasso Hospital, Sikasso, Mali \\ Email: *cis_astou@yahoo.fr
}

How to cite this paper: Cissouma, A., Traoré, M., Dembélé, G., Diarra, S., Keita, A.M., Kissima-Traoré, A., Kassogué, D., Traoré, S.A., Diallo, F., Haidara, D.B.S. and Sow, S. (2021) The Epidemiology of Influenza in Children Hospitalized at the Hospital of Sikasso, Mali 2015-2019. Open Journal of Pediatrics, 11, 460-467. https://doi.org/10.4236/ojped.2021.113043

Received: August 10, 2021

Accepted: September 10, 2021

Published: September 13, 2021

Copyright $\odot 2021$ by author(s) and Scientific Research Publishing Inc. This work is licensed under the Creative Commons Attribution International License (CC BY 4.0)

http://creativecommons.org/licenses/by/4.0/ (c) (i) Open Access

\begin{abstract}
Introduction: Influenza is an acute respiratory infectious disease, highly contagious due to influenza viruses. The objective of this work was to identify, understand the epidemiology of circulating strains and estimate disease transmission. Patients and Methods: The study was carried out in the pediatric department of the Sikasso Hospital. This was a prospective, longitudinal descriptive study over a five-year period (January 1, 2015 to December 31,2019 ). She was interested in severe acute respiratory infections (SARI) for hospitalized patients in the pediatric department. Results: During the study period the prevalence of severe acute respiratory infections among hospitalized children was $21.85 \%$. The majority of cases were observed in 2019 with 58 cases, the sex ratio was 1.8 . The age group from 0 to 1 was the most represented with 100 cases $(48.30 \%)$ followed by 2 to 4 years 73 cases $(35.24 \%)$ and 5 to 15 years 34 cases $(16.46 \%)$. More than half of the patients lived in rural areas $129(62.31 \%)$. Fever and cough were present in the majority of patients. No children had received influenza vaccination. In study 36 (17.39\%) cases were positive for influenza A and B.
\end{abstract}

\section{Keywords}

Sentinel Surveillance, Seasonal Influenza, Sikasso Hospital 


\section{Introduction}

Seasonal influenza is now recognized in children. Its underestimation persists due to the non-specificity of symptoms and the overlap of other winter viral epidemics [1]. According to the World Health Organization (WHO), annual epidemics cause about 3 to 5 million severe cases (requiring hospitalization) and 250,000 to 500,000 deaths per year worldwide [1]. Influenza viruses are characterized by their frequent mutations responsible for the emergence of new viral strains for which populations have little or no immunity and these new strains can in turn be responsible for seasonal epidemics or pandemics that are sometimes very severe and deadly [2]. During annual seasonal epidemics, the infection rate is highest among children, especially school-age children, contributing to the widespread of the influenza virus. The incidence of influenza disease leading to hospitalization is highest in children under 2 years of age [3]. Indeed, the risk of the appearance of particularly virulent pandemic viruses is a haunting for public health actors [2] [3]. As the threat is a matter of concern, measures to combat influenza, including epidemiological surveillance, have been strengthened. Thus, sentinel surveillance, ambulatory surveillance of influenza-like illness and surveillance of severe acute respiratory infections (SARI) in hospitalized patients are recommended by WHO [1]. In the WHO/AFRO region, this surveillance should be done in accordance with the Integrated Disease Surveillance and Response (SIMR) strategy [1]. In order to participate effectively in the global response to the risk of an influenza pandemic, the Ministry of Health of Mali, through the CVD-Mali, is committed to improving influenza surveillance through the creation of sentinel sites. This effort is supported by WHO and the U.S. Government through the Centers for Disease Control and Prevention (CDC) and the Naval Medical Research Unit-3 (NAMRU-3) to enable the implementation of an effective system for the detection of suspected cases and confirmation by state-of-the-art technology (real-time PCR). This sentinel surveillance of influenza at Sikasso Hospital will identify, understand the epidemiology of circulating strains and estimate disease transmission.

\section{Patients and Methods}

The surveillance was carried out in the pediatric department of the Sikasso hospital in Mali. The Sikasso region is the 3rd administrative region of Mali, it is located in the southern part of Mali, with an area of $71,790 \mathrm{~km}^{2}$. It is bounded to the north by the Ségou region, to the south by the Republic of Côte d'Ivoire, to the west by the Republic of Guinea Conakry, to the east by Burkina Faso and to the northwest by the region Koulokoro. This service consists of an outpatient unit, a neonatology unit, a paediatric oncology unit, a general paediatric inpatient unit and an intense nutritional recovery unit (URENI). It has a capacity of 40 beds and receives an average of 2000 admissions per year.

This was a prospective, longitudinal descriptive study over a five-year period (2015 to 2019). 
This surveillance was sentinel on a weekly basis and took place throughout the year, she has been interested in severe acute respiratory infections (SARI) in pediatric hospitalized children. Meeting the case definition: Anyone with a history of fever or fever measured $\geq 38^{\circ} \mathrm{C}$ and respiratory signs (coughing, sneezing, rhinitis, breathing difficulties) with onset in the last ten days. The data collected through an individual notification sheet for each case collected in the sentinel sites from Monday to Thursday were transmitted to the NIC-CVD-Mali at the same time as the samples. Surveillance combined epidemiological information and laboratory results in a single database. This will allow a joint analysis of these two types of data. In parallel with the virological surveillance, epidemiological surveillance was carried out. All patients matching the case definitions were systematically registered and collected. A notification sheet prepared for this purpose has been made available to sentinel sites. It includes the socio-demographic characteristics of the patients, the history, the nature and characteristics of the exposure, the clinical signs, the course of the disease etc. The completed weekly notification form is sent to the National Directorate of Health. All patients meeting the case definition criteria were collected from Monday to Thursday. Sampling, packaging and transport equipment, including swabs, virus transport media (MTV) and notification forms, have been made available to sentinel sites by NIC-CVD-Mali. The operation involves introducing either into a nostril or into the throat a sterile swab and collecting epithelial cells that may contain the viruses by scraping it. The goal is to collect naso and/or oropharyngeal samples in search of respiratory viruses. The samples collected by the clinicians of the sentinel site are stored in the Viral Transport Medium (MTV) in a refrigerator and regularly monitored at a temperature of $+4^{\circ} \mathrm{C}\left(2^{\circ} \mathrm{C}\right.$ to $\left.8^{\circ} \mathrm{C}\right)$. The coolers or insulated boxes containing the samples are sent to the laboratory of the NIC-CVD-Mali twice a week (Tuesday and Thursday) through the carriers contracted with the WHO for the transport of samples of other notifiable diseases. A real-time reverse transcription polymerase chain reaction (RT-PCR) was performed to identify and subtype influenza strains. The samples collected are transported to the reference laboratory. The laboratory receives the samples and checks the acceptability conditions. The samples are placed in a viral transport medium comprising the 2 swabs at a temperature of $2^{\circ} \mathrm{C}$ and $8^{\circ} \mathrm{C}$ in well-closed containers within a given period of 72 hours. Samples are divided into three aliquots, one for CDC, one for CVD-Mali archives and the third for testing. If samples are not tested within 72 hours they are stored at or below $-80^{\circ}$. The real-time PCR technique consists in carrying out a PCR in a single step by using in the reaction medium both primers allowing amplification but also a probe allowing the detection of the PCR products as and when they appear. The probe is labeled with a fluorochrome and fluorescence emission occurs when the probe hybridizes with the target DNA present in the sample. The fluorescence emission is detected at each PCR cycle by the real-time PCR machine; the amount of fluorescence emitted is proportional to the amount of target 
present in the sample. This technology has a triple advantage over the traditional PCR technique: it avoids contamination since the tube containing the PCR products does not need to be opened, it is quantitative and it is very easy to automate. It is also possible to carry out so-called multiplex PCR that is to say that in the same tube several viral genomes are detected thanks to the presence of a mixture of primers and specific probes of several viruses.

\section{Results}

From 2015 to 2019, 9472 patients were hospitalized in the pediatric department of the Sikasso Hospital, including 207 for severe acute respiratory infection (SARI) or $21.85 \%$. We recorded 133 boys (64.25\%) and 74 girls (35.75\%) with a sex ratio of 1.8 compared to Graph 1 . The age group from 0 to 1 was the most represented with 100 cases (48.30\%) followed by 2 to 4 years 73 cases $(35.24 \%)$ and 5 to 15 years 34 cases $(16.46 \%)$ detailed on Graph 2. More than half of the patients lived in rural areas $129(62.31 \%)$ of the cases and 78 (37.69) cases were in urban areas. Graph 3 gives us the repair by year of cases of severe acute respiratory infection, the majority of cases were observed in 2019 with 58 cases followed by 2016 with 43 cases then 2018 with 41 cases and 2015 with 35 cases. More than half of the A and B influence viruses were detected during the cool period, November, December, January and February. Fever and cough were present in the majority of patients with $83.9 \%$ respectively; $90 \%$ followed by runny nose in $65.22 \%$ of the cases detailed in Table 1 . We recorded 4 cases (1.93\%) of chronic condition in children. No patients had received influenza vaccination. In study $36(17.39 \%)$ cases were positive for influenza. ten types of

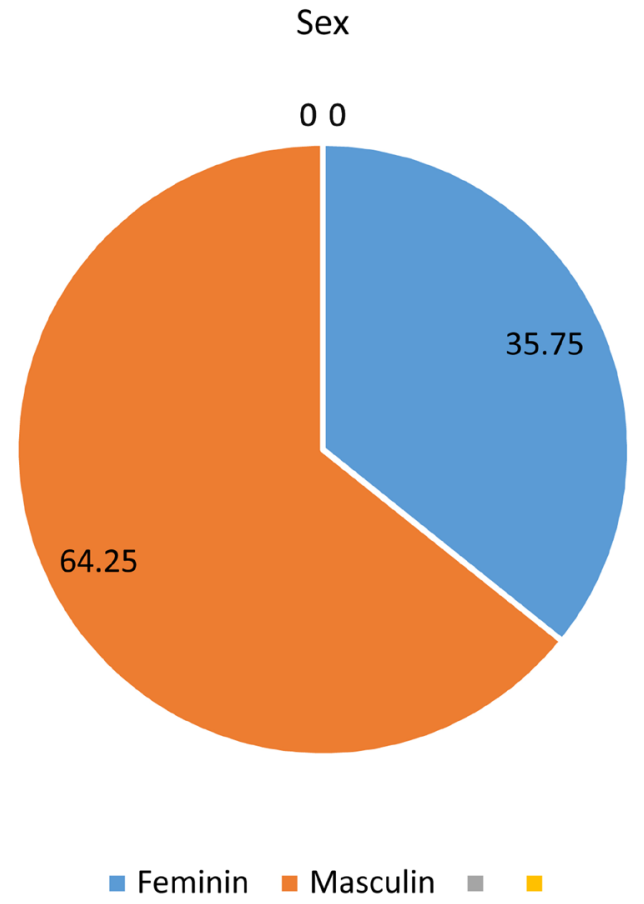

Graph 1. Distribution of cases by sex. 


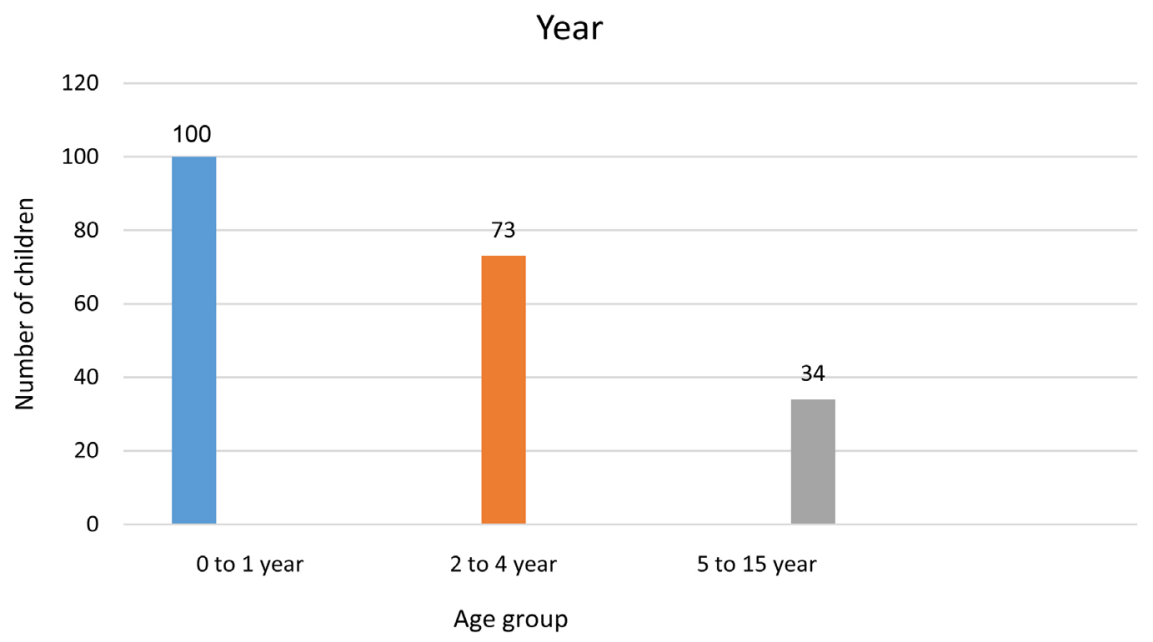

Graph 2. Distribution of cases by age group.

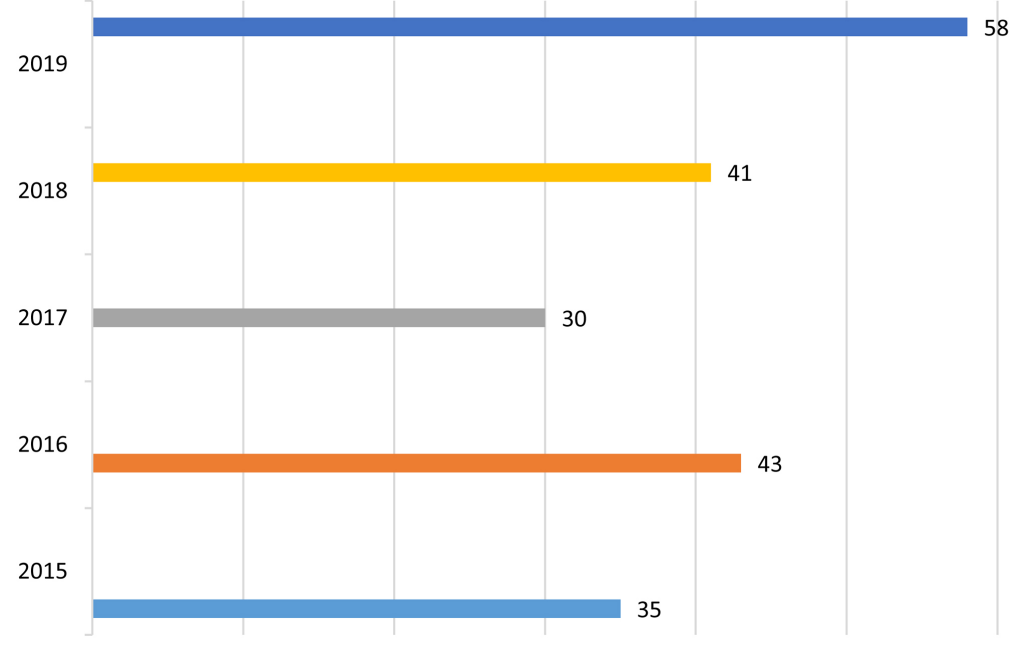

Graph 3. Distribution of cases by year.

Table 1. Distribution of cases by signs/symptoms.

\begin{tabular}{cccc}
\hline Clinical signs & YES & NO & TOTAL \\
\hline History of fever & $57.3 \%$ & $42.7 \%$ & $100 \%$ \\
Fever 38 & $83.09 \%$ & $16.91 \%$ & $100 \%$ \\
Cough & $90 \%$ & $10 \%$ & $100 \%$ \\
Sore throat & $46.7 \%$ & $53.3 \%$ & $100 \%$ \\
Nasal flow & $34.78 \%$ & $65.22 \%$ & $100 \%$ \\
Difficulty breathing & $48.31 \%$ & $51.69 \%$ & $100 \%$ \\
Danger signs & $35.55 \%$ & $65.45 \%$ & $100 \%$ \\
Stridor & $10.5 \%$ & $89.5 \%$ & $100 \%$ \\
\hline
\end{tabular}

influenza B (2 B and 2 B Victoria), 26 types of influenza A (19 A/H1N1 pandemic, 7 A/H3N2) (Graph 4). We did not record cases of avian influenza and the lethality of influenza-positive cases was $1.2 \%$. 


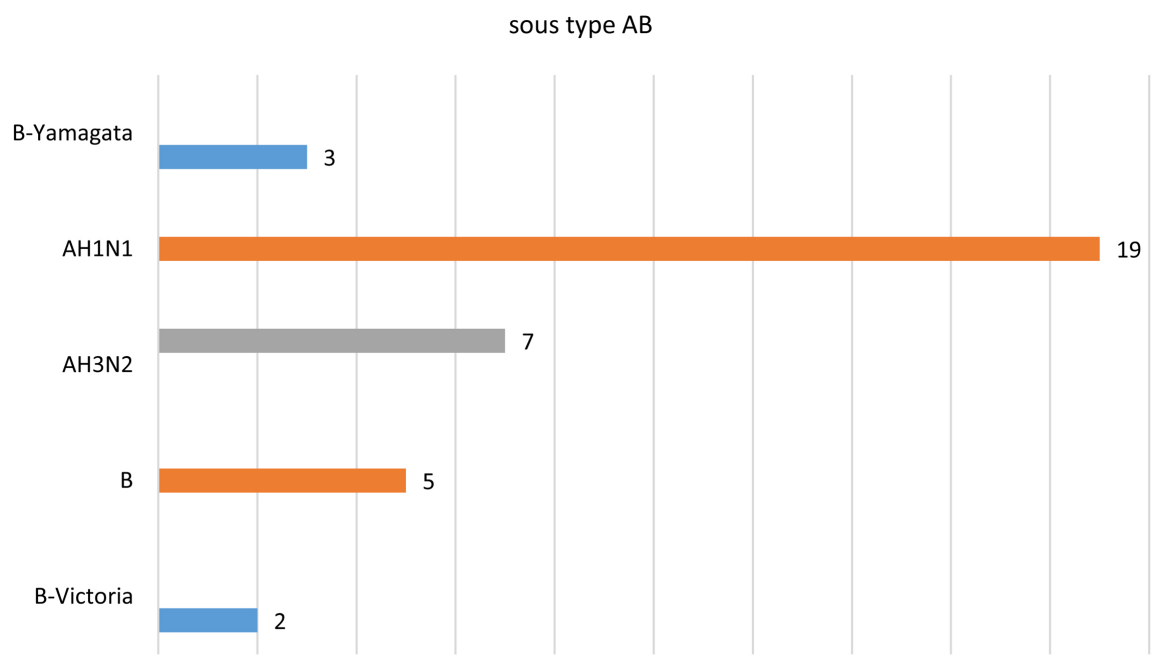

Graph 4. Distribution of positive cases by subtype AB.

\section{Discussion}

In temperate climates, seasonal epidemics occur mainly during the winter, while tropical and subtropical regions, influenza can appear throughout the year, with more irregular outbreaks [4]. Hence the need for annual surveillance of cases of influenza-like illness and SARI. In the tropics and subtropics, data on the burden and impact of influenza are limited. However, there is growing evidence that the burden of influenza may be significant and probably comparable to the impact of the disease on health in developed countries [4] [5]. Influenza is a winter disease: In this study, $40 \%$ of influenza A and B viruses are isolated in January and February, compared to 25\% in November and December, and $8 \%$ in March and April. The annual attack rate is estimated at $5 \%-10 \%$ in adults and $20 \%-30 \%$ in children [5]. During this study, children under two contributed to the high rate of transmission. There are three types of seasonal influenza named: A, B, and $\mathrm{C}$, of decreasing importance. The epidemic most often combines the two types $\mathrm{A}$ and $\mathrm{B}$ of viruses. These viruses belong to the family Orthomyxoviridae [6]. Influenza A viruses are subdivided into subtypes according to the different kinds and associations of surface proteins of the virus. Among the many subtypes of influenza viruses, subtypes $\mathrm{A} / \mathrm{H} 1 \mathrm{~N} 1$ and $\mathrm{A} / \mathrm{H} 3 \mathrm{~N} 2$ are currently circulating in men [7]. Circulating influenza $B$ viruses can be divided into two main groups, or lineages, called the $\mathrm{B} / \mathrm{Yamagata}$ and $\mathrm{B} / \mathrm{Victoria}$ lines. Influenza $\mathrm{B}$ viruses are not classified as subtypes [7]. The influenza $C$ virus is only very rarely detected and generally causes only mild infections, so its impact on public health is of lesser importance [6] [7]. A and B were detected in the study. The child is a prime target for influenza virus infections. He is very exposed because he has the first contact with the viruses of this family, and the community lifestyle of nurseries and schools facilitates contamination. A French study shows that the highest rate of seasonal influenza attack is observed in school-aged children [8]. In Finland, it has been shown that the seasonal influenza attack rate can reach $30 \%$ of children between five and 14 years of age, and in the United States, the 
highest rates of influenza hospitalizations are observed in children under four years of age and those over 65 years of age [9] [10]. Seasonal influenza is usually characterized by the sudden onset of fever, headache, myalgia, dry cough, sore throat and rhinitis [11]. In a population of infected and non-hospitalized patients, the positive predictive value of the presence of fever, cough and sudden onset does not exceed 30\% [12]. In subjects over 65 years of age, carriers of chronic pathologies and hospitalized, this value reaches $53 \%$ on the criteria of fever, cough and a disease of less than seven days [13]. The identification of influenza A or B virus infection is particularly useful in the pre- or post-epidemic period. It is essential in all subjects hospitalized for respiratory syndrome or who have consulted for influenza-like illness. There are many tools allowing a direct search for the virus or its constituents in respiratory secretions: isolation of the virus in culture, The isolation of influenza viruses in culture on embryonated chicken egg or on mammalian cells cultivated in vitro is still today the reference method for the identification of influenza viruses [14], the detection of antigens by immunofluorescence (IF) or immunoenzymatic (IEA) have been developed by several virology laboratories for the search for influenza viruses. Most often, the technique is the same, and the differences relate to the preparation of the antigen and the type of antibodies [15], and the search for RNA by RT-PCR is particularly interesting to detect influenza virus infection when samples are taken more than four days after infection [16]. The PCR technique was used for analysis of our samples at the National Reference Laboratory, Samples of suspected cases of $\mathrm{H} 5 \mathrm{~N} 1$ and undetermined samples will be immediately sent to a WHO reference laboratory (NAMRU-3) or to a WHO collaborating center (CDC-Atlanta, etc.) for further analysis.

\section{Conclusion}

Five years of sentinel surveillance of seasonal influenza have shown that influenza is a public health problem in the Sikasso region of Mali. Vaccination would be a useful prevention strategy.

\section{Acknowledgements}

We would like to thank CDC-Atlanta for the influenza surveillance cooperative agreement through with this work has been possible.

\section{Conflicts of Interest}

No conflict of interest.

\section{References}

[1] World Health Organization (WHO) (2013) Fact Sheet 211: Influenza (Seasonal). World Health Organization. https://www.who.int/en/news-room/fact-sheets/detail/influenza-(seasonal)

[2] Gouarin, C., Vabret, A., Dina, J., Petitjean, J., Brouard, J., Cuvillon-Nimal, D., et al. (2008) Study of Influenza C Virus Infection in France. Journal of Medical Virology, 
80, 1441-1446. https://doi.org/10.1002/jmv.21218

[3] Centers for Disease Control and Prevention (CDC) (2005) Surveillance for Laboratory-Confirmed, Influenza-Associated Hospitalizations-Colorado, 2004-05 Influenza Season. Morbidity and Mortality Weekly Report, 54, 535-537.

[4] Thompson, W.W., Weintraub, E., Dhankhar, P., Cheng, O.Y., Brammer, L., Meltzer, M.I., et al. (2009) Estimates of US Influenza-Associated Deaths Made Using Four Different Methods. Influenza and Other Respiratory Viruses, 3, 37-49. https://doi.org/10.1111/j.1750-2659.2009.00073.x

[5] Nair, H., Abbulah Brooks, W., Katz, M., et al. (2011) Global Burben of Repiratory Infections Due to Seasonal Influenza in Young Children: A Systematic Review and Meta-Analysis. Lancet, 378, 1917-1913.

[6] Gouarin, C., Vabret, A., Dina, J., Petitjean, J., Brouard, J., Cuvillon-Nimal, D., et al. (2008) Study of Influenza C Virus Infection in France. Journal of Medical Virology, 80, 1441-1446. https://doi.org/10.1002/jmv.21218

[7] Manuguerra, J.C. (2001) Ecology, Biodiversity and Evolution of Influenza Viruses. Virology, 5, 195-205.

[8] Carrat, F., Flahault, A., Boussard, E., Ferran, N., Dangoumau, L. and Valleron, A.J. (1998) Surveillance of Influenza-Like Illness in France: The Example of the 1995/1996 Epidemic. Journal of Epidemiology and Community Health, 52, S32-S38.

[9] Heikkinen, T. (2006) Influenza in Children. Acta Pediatrica, 95, 778-784. https://doi.org/10.1080/08035250600612272

[10] Glezen, W.P., Decker, M. and Perrotta, D.M. (1987) Survey of Underlying Conditions of Persons Hospitalized with Acute Respiratory Disease during Epidemics in Houston, 1978-1981. The American Review of Respiratory Disease, 136, 550-555. https://doi.org/10.1164/ajrccm/136.3.550

[11] Nicholson, K.G. (1992) Clinical Features of Influenza. Seminars in Respiratory Infections, 7, 26-37.

[12] Govaert, T.M., Dinant, G.J., Aretz, K. and Knottnerus, J.A. (1998) The Predictive Value of Influenza Symptomatology in Elderly Poeple. Family Practice, 15, 16-22. https://doi.org/10.1093/fampra/15.1.16

[13] Walsh, E.E., Cox, C. and Falsey, A.R. (2002) Clinical Features of Influenza a Infection in Older Hospitalized Persons. Journal of the American Geriatrics Society, 50, 1498-1503. https://doi.org/10.1046/j.1532-5415.2002.50404.x

[14] Chomel, J.J., Remilleux, M.F., Marchand, P. and Aymard, M. (1992) Rapid Diagnosis of Influenza A Comparison with Immunocapture and Culture. Journal of Virological Methods, 37, 337-344. https://doi.org/10.1016/0166-0934(92)90033-A

[15] Grandien, M., Pettersson, C.E., Gardner, P.S., Linde, A. and Stanton, A. (1985) Rapid Viral Diagnosis of Acute Respiratory Infections: Comparison of Enzyme-Linked Immunosorbent Assay and the Immunofluorescence Technique for Detection of Viral Antigens in Nasopharyngeal Secretions. Journal of Clinical Microbiology, 22, 757-760. https://doi.org/10.1128/jcm.22.5.757-760.1985

[16] Donofrio, J.C., Coonrod, J.D., Davidson, J.N. and Betts, R.F. (1992) Detection of Influenza A and B in Respiratory Secretions with the Polymerase Chain Reaction. PCR Methods and Applications, 1, 263-268. https://doi.org/10.1101/gr.1.4.263 